\title{
The Premonitory Urge for Tics Scale in a large sample of children and adolescents: psychometric properties in a developmental context. An EMTICS study
}

\author{
Thaïra J. C. Openneer ${ }^{1}\left[\right.$ C Zsanett Tárnok ${ }^{2} \cdot$ Emese Bognar $^{2} \cdot$ Noa Benaroya-Milshtein ${ }^{3} \cdot$ Blanca Garcia-Delgar $^{4}$. \\ Astrid Morer ${ }^{4,5,6} \cdot$ Tamar Steinberg $^{3} \cdot$ Pieter J. Hoekstra ${ }^{1} \cdot$ Andrea Dietrich $^{1} \cdot$ and the EMTICS collaborative group
}

Received: 18 April 2019 / Accepted: 22 November 2019 / Published online: 4 December 2019

(C) The Author(s) 2019

\begin{abstract}
Premonitory urges are uncomfortable physical sensations preceding tics that occur in most individuals with a chronic tic disorder. The Premonitory Urge for Tics Scale (PUTS) is the most frequently used self-report measure to assess the severity of premonitory urges. We aimed to evaluate the psychometric properties of the PUTS in the largest sample size to date $(n=656)$, in children aged 3-16 years, from the baseline measurement of the longitudinal European Multicenter Tics in Children Study (EMTICS). Our psychometric evaluation was done in three age-groups: children aged 3-7 years $(n=103)$, children between 8 and 10 years $(n=253)$, and children aged $11-16$ years $(n=300)$. The PUTS exhibited good internal reliability in children and adolescents, also under the age of 10 , which is younger than previously thought. We observed significant but small correlations between the severity of urges and severity of tics and obsessive-compulsive symptoms, and between severity of urges and ratings of attention-deficit/hyperactivity disorder and internalizing and externalizing behaviors, however, only in children of 8-10 years. Consistent with previous results, the 10th item of the PUTS correlated less with the rest of the scale compared to the other items and, therefore, should not be used as part of the questionnaire. We found a two-factor structure of the PUTS in children of 11 years and older, distinguishing between sensory phenomena related to tics, and mental phenomena as often found in obsessive-compulsive disorder. The age-related differences observed in this study may indicate the need for the development of an age-specific questionnaire to assess premonitory urges.
\end{abstract}

Keywords Tourette syndrome · Premonitory urges · Premonitory Urge for Tics Scale (PUTS) · Psychometric properties · Obsessive-compulsive symptoms

Pieter J. Hoekstra and Andrea Dietrich contributed equally to this work.

Electronic supplementary material The online version of this article (https://doi.org/10.1007/s00787-019-01450-1) contains supplementary material, which is available to authorized users.

Thaïra J. C. Openneer

t.openneer@accare.nl

1 Department of Child and Adolescent Psychiatry, University of Groningen, University Medical Center Groningen, Hanzeplein 1 XA10, 9713 GZ Groningen, The Netherlands

2 Vadaskert Child and Adolescent Psychiatric Hospital, Budapest, Hungary

3 Child and Adolescent Psychiatry Department, Affiliated to Sackler Faculty of Medicine, Schneider Children's Medical Center of Israel, Tel Aviv University, Petah-Tikva, Israel

\section{Introduction}

Chronic tic disorders, i.e. Tourette syndrome (TS) and persistent (chronic) motor or vocal tic disorder, are childhoodonset disorders characterized by the presence of multiple

4 Department of Child and Adolescent Psychiatry and Psychology, Institute of Neurosciences, Hospital Clinic Universitari, Barcelona, Spain

5 Institut d'Investigacions Biomediques August Pi i Sunyer (IDIBAPS), Barcelona, Spain

6 Centro de Investigacion en Red de Salud Mental (CIBERSAM), Instituto Carlos III, Madrid, Spain 
motor and/or vocal tics for at least 1 year [1]. Tic disorders are often accompanied by other disorders, particularly obsessive-compulsive disorder (OCD) and attention-deficit/ hyperactivity disorder (ADHD), but also autism spectrum disorder (ASD) and internalizing problems (i.e. anxiety or depression) [2].

Up to $93 \%$ of individuals with TS experience an uncomfortable physical sensation preceding their tics, known as a premonitory urge [3]. Two broad types of premonitory urges have been reported: sensory feelings such as an 'itch' or 'pressure' in certain bodily areas, or mental phenomena such as 'the feeling that something is not "just right" or complete' $[4,5]$. Premonitory urges are often reported to be even more distressing and impairing than tics themselves $[6,7]$ and are an important target for behavioral therapy $[8,9]$, as they may facilitate suppression of the impending tic. In recent years, our understanding of the premonitory urge in TS has rapidly expanded (see for a review [4]), providing more knowledge about the role of premonitory urges in TS. For example, the level of interoceptive awareness proved to be one of the stronger predictors of premonitory urges in TS [43].

Despite the recent advances in our understanding of the role of premonitory urges in TS, there is still much uncertainty about the age of onset and development of premonitory urges across childhood and adolescence. For instance, while tics typically start around the age of 6-7 years, it has been assumed that children do not become aware of their premonitory urges until on average 3 years after tic onset $[3,10]$. This suggests that premonitory urges may not be present at the onset of TS, but may develop later [11, 12]. In addition, it has been thought that young children are less consistent in reporting their awareness of premonitory urges before the age of 10 years [12]. However, a recent large study found that premonitory urges were reported in $46.7 \%$ of the children with TS younger than 10 years, thus suggesting that premonitory urges may be experienced at a younger age than previously thought and, furthermore, that children under the age of 10 may be able to reliably report their premonitory urges [14].

The Premonitory Urge for Tics Scale (PUTS [12]) is the most frequently used self-report measure to assess the severity of premonitory urges. Studies investigating the psychometric properties of the PUTS have so far indicated a good internal reliability and correlations with the Yale Global Tic Severity Scale (YGTSS [15]) for children of 11 years and older, but not for younger children [12, 16, 17]. Similarly, PUTS scores of children aged 11 years and older (and not younger children) correlated well with the Children's YaleBrown Obsessive Compulsive Scale (CY-BOCS [18]), which might not be surprising given that some premonitory urges (i.e. "the feeling that something is not "just right" or not complete') have been shown to be related to OCD symptoms [5]. Thus, while studies so far observed good psychometric properties of the PUTS in children of 11 years and older [12, $16,17]$, the suitability of the PUTS for younger children has not yet been established, even though premonitory urges may already be present at a young age.

The PUTS was originally designed as a one-dimensional measure [12]. However, a two- to three-factor [16, 19] solution emerged from recent factor analyses in adolescents and adults; one factor broadly represented mental urges, including the aforementioned OCD-related premonitory urges, i.e. "the feeling that something is not "just right" or not complete' [19], while the second factor reflected the intensity or frequency of the urges [16]. Yet, given that the typical course of TS is characterized by a symptomatic peak in early adolescence and decline into adulthood [20], findings from adolescents and adults may not hold true for younger children. Furthermore, existing studies examining the psychometric properties of the PUTS in children and adolescents are hampered by small sample sizes $(n=40$ to $n=82$; [12, $16,17])$, which made it difficult to investigate age-related differences in the psychometric properties of the PUTS across childhood and adolescence.

The aim of the present study, therefore, was to examine the psychometric properties of the PUTS in a large sample of 656 children, aged 3-16 years (of which 356 children were below 11 years) from an European multicenter study. We aimed to replicate previous work $[12,16,17]$ and to further investigate the psychometric properties in young children. First, we investigated the internal consistency of the PUTS. Second, we assessed correlations with tic and OCD severity, also exploring the influence of two OCD-related items of the PUTS. Third, we looked into associations of the PUTS with other comorbid symptom domains (i.e. ADHD, oppositional defiant disorder [ODD], ASD, and externalizing and internalizing symptoms), given the previous inconsistent literature in small samples $[5,12,16,21]$. Finally, to extend earlier work $[16,19]$ we conducted a factor analysis of the PUTS in the whole sample and in three different age groups.

\section{Methods}

\section{Participants}

Our study sample consisted of 656 3-16 years old children and adolescents with a chronic tic disorder participating in the baseline measurement of the longitudinal European Multicenter Tics in Children Study (EMTICS). EMTICS aims to identify the role of genes, autoimmunity, and psychosocial stress on the onset and course of tics (see for a more detailed description: [22]). Participants were recruited from 16 child and adolescent psychiatry or pediatric neurology outpatient clinics, or through advertisement of the study to patient organizations and 
other health professionals. Exclusion criteria were having a serious medical illness, treatment with antibiotics during the last month (as the included children were also eligible to participate in a separate antibiotic study [see $22]$ ), or an inability to understand and comply with the study procedures. The adolescent's parents or legal guardians provided written informed consent and the participating adolescent provided written consent or assent in line with the local medical-ethical regulations. The study was approved by the local research ethics committee of the participating centers.

\section{Procedures}

Children and adolescents were asked to complete questionnaires on premonitory urges and symptoms of ADHD, ODD, ASD, and internalizing and externalizing disorders within 2 weeks before the baseline visit, and to bring these to the first visit. During the baseline visit a trained study clinician assigned a clinical diagnosis of a chronic tic disorder, OCD, and/or ADHD according to DSM-IV-TR criteria [13], and rated the severity of tics and OCD with well-validated measures (see further below).

\section{Measures}

\section{Premonitory Urge for Tics Scale (PUTS)}

The PUTS was developed by Woods et al. [12] and has previously been demonstrated as having good internal reliability, temporal stability, and correlations with the YGTSS and CY-BOCS in children of 11 years and older and in adults [12, 16, 17, 19, 23]. It measures sensory and mental phenomena associated with premonitory urges in 10 items on a four-point scale (range 10-40). The first 6 items include itchiness, energy, pressure, tense feeling, incomplete, or a not "just right" feeling before performing a tic. The additional 4 items assess whether these feelings are experienced almost all the time before a tic, if they happen with every tic, if they go away after the tic is performed, and if subjects are able to stop the tics for a short period of time. Woods et al. [12] noted that the 10th item had a lower correlation with the rest of the scale compared to the other items. As a result, some studies using the PUTS omit the 10th item in favor of a 9-item scale (e.g., [17]. In the present study, the 10-item PUTS was administered to participants to replicate the data analysis of Woods et al. [12] (i.e., to determine how the 10th item correlated with the rest of the scale using a larger sample size). A higher total score indicates more severe premonitory urges.
Yale Global Tic Severity Scale (YGTSS)

The YGTSS [15] (Cronbach's alpha in our study $\alpha=0.87$ ) is a semi-structured clinician-rated instrument that evaluates the severity of tics across five dimensions each scored on a five-point scale, by assessing the number, frequency, intensity, complexity, and interference of, respectively, motor and vocal tics during the past week. A total tic severity score can be obtained (range $0-50$ ), and also severity scores for vocal tics (range $0-25, \alpha=0.85$ ) and motor tics (range $0-25$, $\alpha=0.89$ ) by summing up the respective scores. A higher total, vocal, or motor score indicates more severe tics.

\section{Children's Yale-Brown Obsessive-Compulsive Scale (CY-BOCS)}

The CY-BOCS is a clinician-administered semi-structured interview developed to assess the severity of obsessions and compulsions in children $[18,24]$ (Cronbach's alpha in our study $\alpha=0.93$ ). The symptoms are evaluated across five areas, including the time, interference, distressing nature, resistance, and control associated with obsessions and compulsions during the past week on a five-point scale. Besides a total OCD severity score (range $0-40$ ), a severity score was obtained for obsessions (range $0-20 ; \alpha=0.92$ ) and compulsions (range $0-20 ; \alpha=0.94$ ). A higher score indicates higher severity ratings.

\section{Other symptom domains}

To assess ADHD and ODD symptom severity, we used the parent-rated Swanson Nolan and Pelham-IV rating scale (SNAP-IV [25, 26]). To investigate ASD severity, we used the parent-rated Autism Spectrum Screening Questionnaire (ASSQ [27]), while the Strengths and Difficulties Questionnaire (SDQ [28]) was used to assess broadband internalizing and externalizing symptom severity. See Supplement 1 for more information about these questionnaires.

\section{Data analytic strategy}

Prior to analysis, we removed outliers $(\geq|3.0|$ standard deviations from the mean; up to $0.9 \%$ ). We checked on the normal distribution of the residues, and used log-transformation to normalize scale scores where appropriate (i.e., only for the total severity score of the CY-BOCS, leading to a normal distribution). Then, site differences were removed by regressing out the effect of site variance from each measure and the saved residuals were added to each score of the respective variable that was used for analysis.

We distinguished three age groups: children $\leq 7$ years $(n=103)$, children between 8 and 10 years $(n=253)$, and children $\geq 11$ years $(n=300)$. As a supplementary analysis 
to allow for comparisons with the existing literature [12, $16,17]$, we also divided our sample into two age groups; children $\leq 10$ years, $(n=356)$ and children and adolescents $\geq 11$ years, $(n=300)$.

Between-group characteristics were tested with the nonparametric Kruskal-Wallis H test (as sex was non-normally distributed), a Chi-square $\left(\chi^{2}\right)$ test, and an analysis of variance (ANOVA), with a Bonferroni correction for multiple comparisons. Differences in the means of the PUTS total score and individual PUTS items between different age groups were also tested with a Bonferroni-corrected ANOVA. For each age group, the Cronbach's alpha $(\alpha)$ was first calculated for the 10 PUTS items, and additionally for the 9-item PUTS omitting the 10th item to determine internal reliability. In addition, the item-total correlation (i.e. the correlation between each individual item and the remaining items) was evaluated by Pearson's product-moment correlation coefficients $(r) ; r$ values $>0.20$ were considered satisfactory [29]. In addition, the Cronbach's $\alpha$ was calculated over the remaining items (thus, without the initial individual items). A Cronbach's $\alpha$ value of around 0.7 was considered acceptable, of 0.8 good, and of 0.9 excellent [30].

To examine the correlations between the PUTS and tic and OCD severity, Pearson product-moment correlations were computed. We additionally explored correlations of the PUTS with symptom severity of ADHD, ODD, ASD, and internalizing and externalizing symptoms. Effect sizes between 0.1 and 0.3 were considered low, between 0.3 and 0.5 moderate, and those over 0.5 high [31].

Furthermore, the underlying factor structure of the PUTS was investigated by conducting a principal axis exploratory factor analysis (EFA). We used direct oblimin rotation, as we assumed that possible factors would be correlated in line with a previous study [16], first, for the total group, and then for different age groups. The factorability of the data (i.e. the assumption that there are correlations amongst items so that coherent factors can be identified), was tested by looking at the inter-item correlations and measures of sampling adequacy. Ideally, an inter-item correlation matrix is considered factorable when the majority of the correlation coefficients computed are in the moderate range; i.e. $r$ values between .20 and .80 [32]. If an item produced a significant number (two or more) inter-item correlations below .20 (i.e., items are not representing the same construct) or above .80 (i.e., multicollinearity), the items were removed before conducting the EFA $[32,33]$. The adequacy of the sampling for the factor analysis with the remaining items was estimated with the Kaiser-Meyer-Olkin (KMO) statistic; its values range from 0 to 1 . KMO values greater than 0.6 represent acceptable sampling adequacy [34]. In addition, Bartlett's test of sphericity was used to assess the suitability of the data for structure detection: a significant test indicates that the individual variables are sufficiently correlated for a factor analysis to be performed. As an outcome measure, we looked at the communalities, representing the proportion of the variance that can be accounted for by the extracted factors. Number of factors were determined by the scree plot and eigenvalues $>1$ [33]. Low communality scores $<0.02$ may indicate that there are additional factors, which thus should be removed from the current factor [32].

Finally, as a sensitivity analysis, we re-analyzed the correlations between the PUTS and CY-BOCS and the factor analyses without the two OCD-related items (i.e. items 4 and 5: "the feeling that something is not "just right" or not complete'), and repeated all analyses without excluding outliers. All statistical analyses were performed using SPSS version 23 (SPSS Inc. USA), using a significance level of $p<0.05$.

\section{Results}

\section{Group characteristics}

See Table 1 for the group characteristics. The mean age for tic onset in the total sample was 6 years. Children aged $\leq 7$ years experienced the least amount of urges (81\%), whereas children aged $\geq 11$ years reported the most urges $(97.5 \%)$. All age groups differed significantly from each other in PUTS severity; children $\leq 7$ years had the lowest PUTS severity score, and children $\geq 11$ years the highest score. Children $\geq 11$ years had higher tic severity as measured by the YGTSS compared to children of $\leq 7$ years, but not to children 8-10 years. There were no significant age group differences in sex, OCD severity, or presence of comorbid OCD or ADHD diagnoses, although comorbid OCD and ADHD diagnoses increased (non-significantly) across age.

\section{Item-by-item frequencies of the PUTS}

Table 2 shows that the group of children $\geq 11$ years had the highest mean scores on most items of the PUTS, the children between 8 and 10 years scored intermediate, and the youngest group ( $\leq 7$ years) scored lowest. Likewise, in the two-group analysis, children $\geq 11$ years had higher mean PUTS scores per individual PUTS item compared to children $\leq 10$ years, except for item 1 and 4 (see Supplementary Table S2a).

See Fig. 1 for item-by-item response frequencies of the PUTS for children in the three age groups. Items 1-3 were on average reported by $20 \%$ of the children $\leq 7$ years, $30 \%$ of children $8-10$ years, and $40 \%$ of children $\geq 11$ years. The most commonly endorsed sensations in all groups were items $6-10$, from $40 \%$ of the children $\leq 7$ years to $70 \%$ of the children $\geq 11$ years. The OCD-related urges 'feelings of something being not "just right" or not complete' (items 


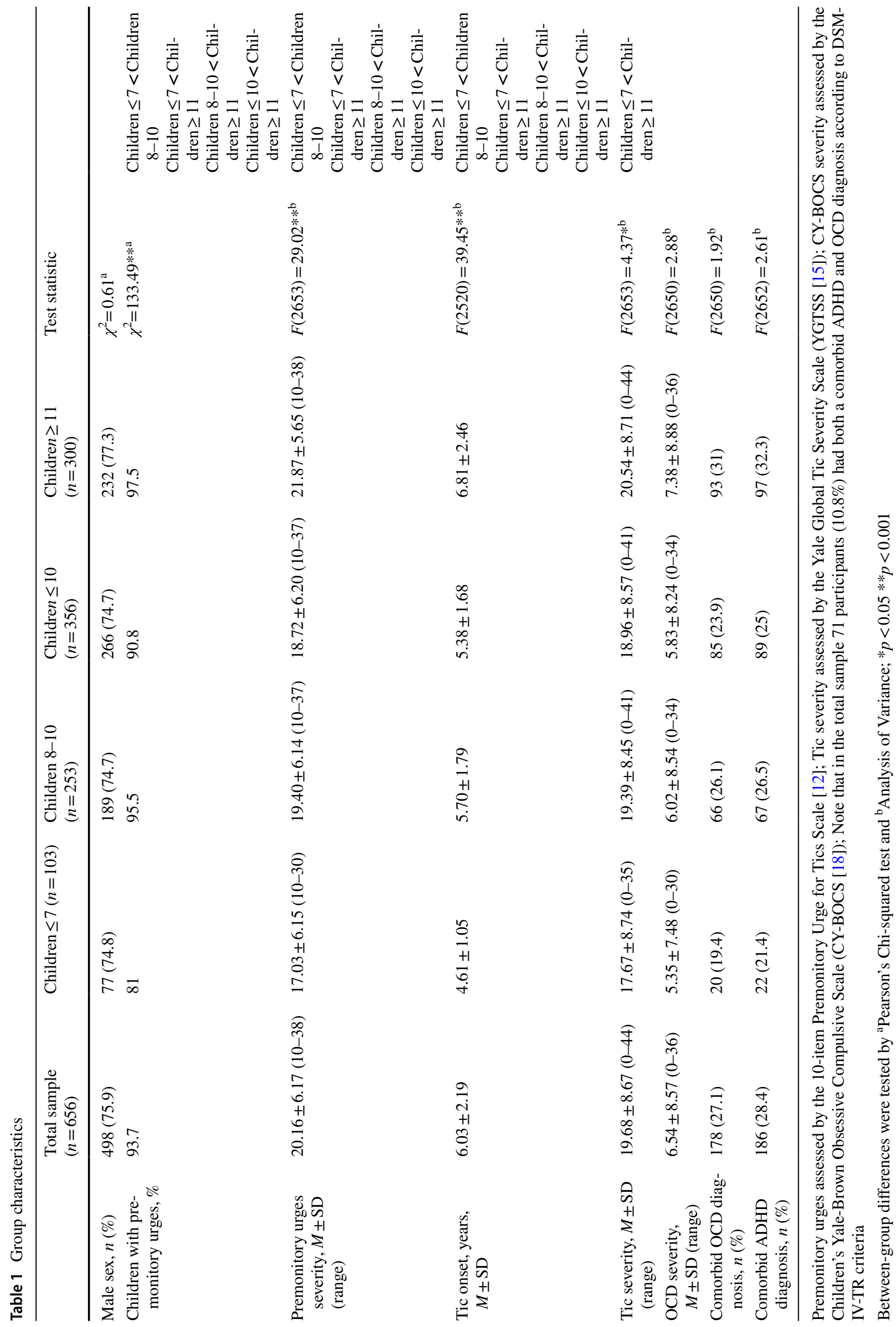




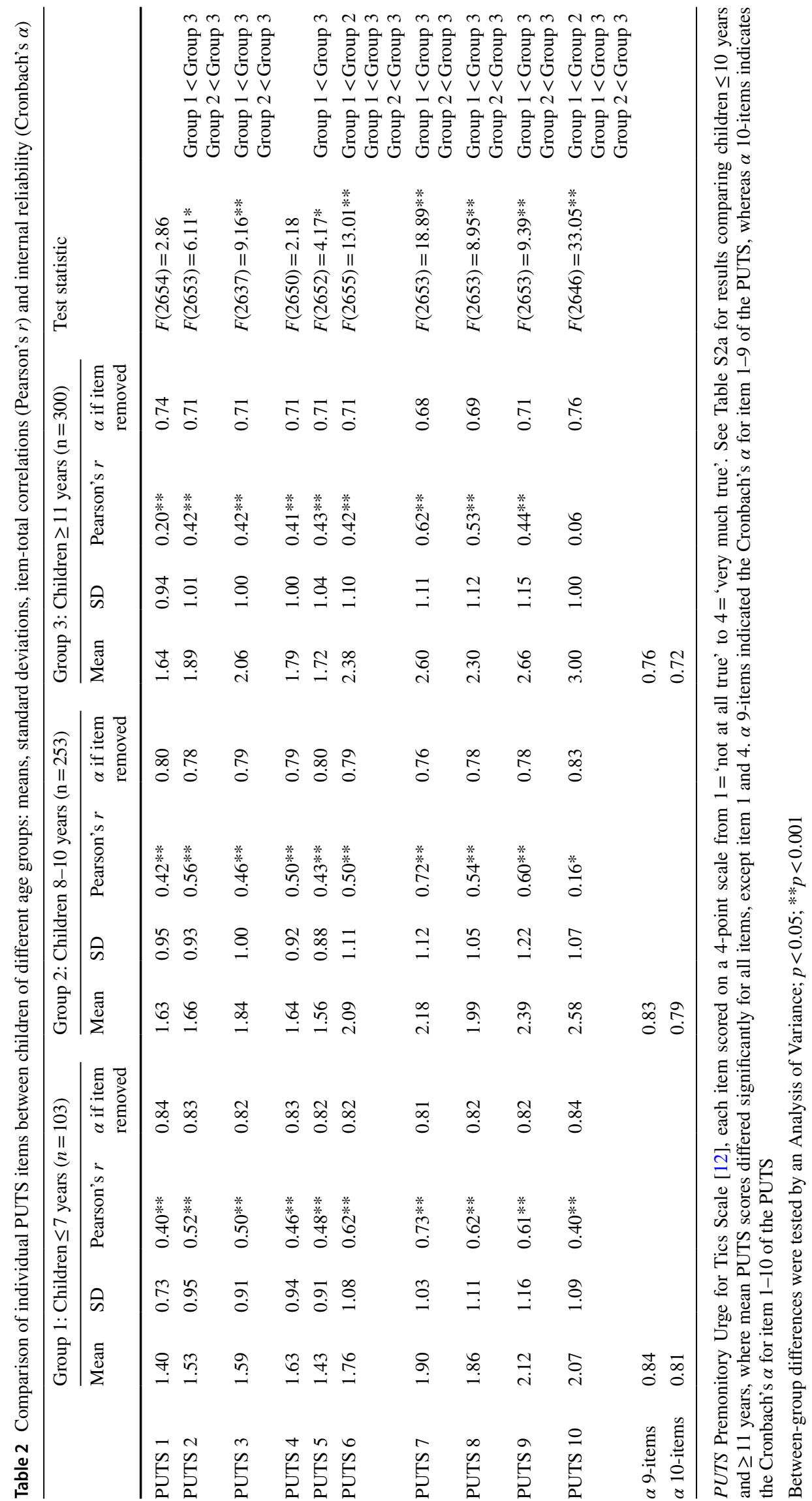




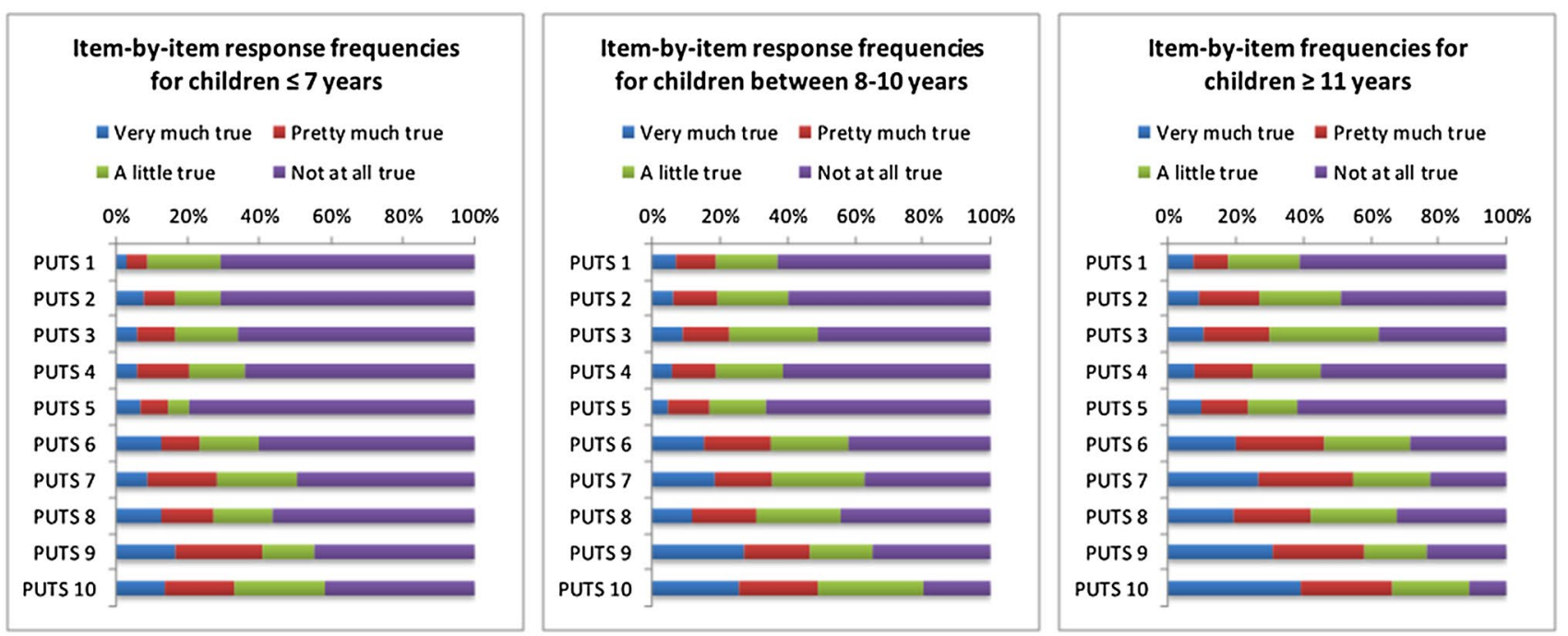

PUTS, Premonitory Urge for Tics Scale item [12], each item scored on a 4-point scale from $1=$ 'not at all true' to $4=$ ' 'very much truc'.

Fig. 1 Item-by-item response frequencies of premonitory urges for children of 7 years and younger, children between 8 and 10 years and children of 11 years and older

4 and 5) were endorsed by almost $40 \%$ and $20 \%$ of children $\leq 7$ years; $40 \%$ and $30 \%$ of children between 8 and 10 ; and $45 \%$ and $40 \%$ children $\geq 11$ years, respectively.

\section{Means, standard deviations, internal reliability}

Table 2 presents the Cronbach's $\alpha$ for each PUTS item across the three age groups after removal of the respective item. Consistent with the decision of previous authors $[12,16]$ to remove item 10 from further analyses, the results showed a lower correlation of item 10 with the rest of the scale for all age groups relative to the other items. Furthermore, the Cronbach's $\alpha$ was similar or higher for all age groups after omitting the 10th item. Therefore, the subsequent analyses were done with the first 9 items of the PUTS. Thus, for the total sample of 656 children, the Cronbach's $\alpha$ for the 9-item PUTS was .80 ( $\alpha=0.78$ for the 10-item PUTS), representing good internal reliability. (See Supplementary Table S2a for the Cronbach's $\alpha$ for each PUTS item in the two-group analysis).

\section{Associations of the PUTS with the YGTSS and CY-BOCS}

For the total sample of 656 children, we observed significant but small positive correlations between the PUTS and the YGTSS total score and all subscales (see Table 3). After analyzing the three age groups, we found that children aged 8-10 years old drove the significant correlations, but not younger or older children. Similarly, in the two-group analysis, significant correlations were present only in children $\leq 10$ years and not in children $\geq 11$ years (Supplementary Table S2b).

A similar pattern appeared for CY-BOCS subscale scores with small but significant positive correlations with the PUTS in the total sample, which were again driven by children aged 8-10 years. Although the CY-BOCS obsession and compulsion subscales reached statistical significance, correlations with the CY-BOCS total score did not (see Table 3 for the results of the three-group analysis, and Supplementary Table S2b for the results of the two-group analysis).

After removing the two items that are often associated with OCD symptomatology in the three age groups; i.e. 'the feeling that something is not "just right" and "the feeling that something is not complete' (items 4 and 5), the significant correlations between PUTS severity and OCD severity disappeared for the obsessions-subscale and diminished for the compulsions-subscale (Supplementary Table S2c).

\section{Associations of the PUTS with other symptom domains}

A similar age-related pattern was observed after correlating the PUTS total score with scores for ASD, ADHD, ODD, and internalizing and externalizing behaviors for the total sample and for the three age groups (see Supplementary Table S2d). Significant positive, yet weak, correlations between the PUTS total score and measures for ADHD, internalizing, and externalizing behaviors were only present 


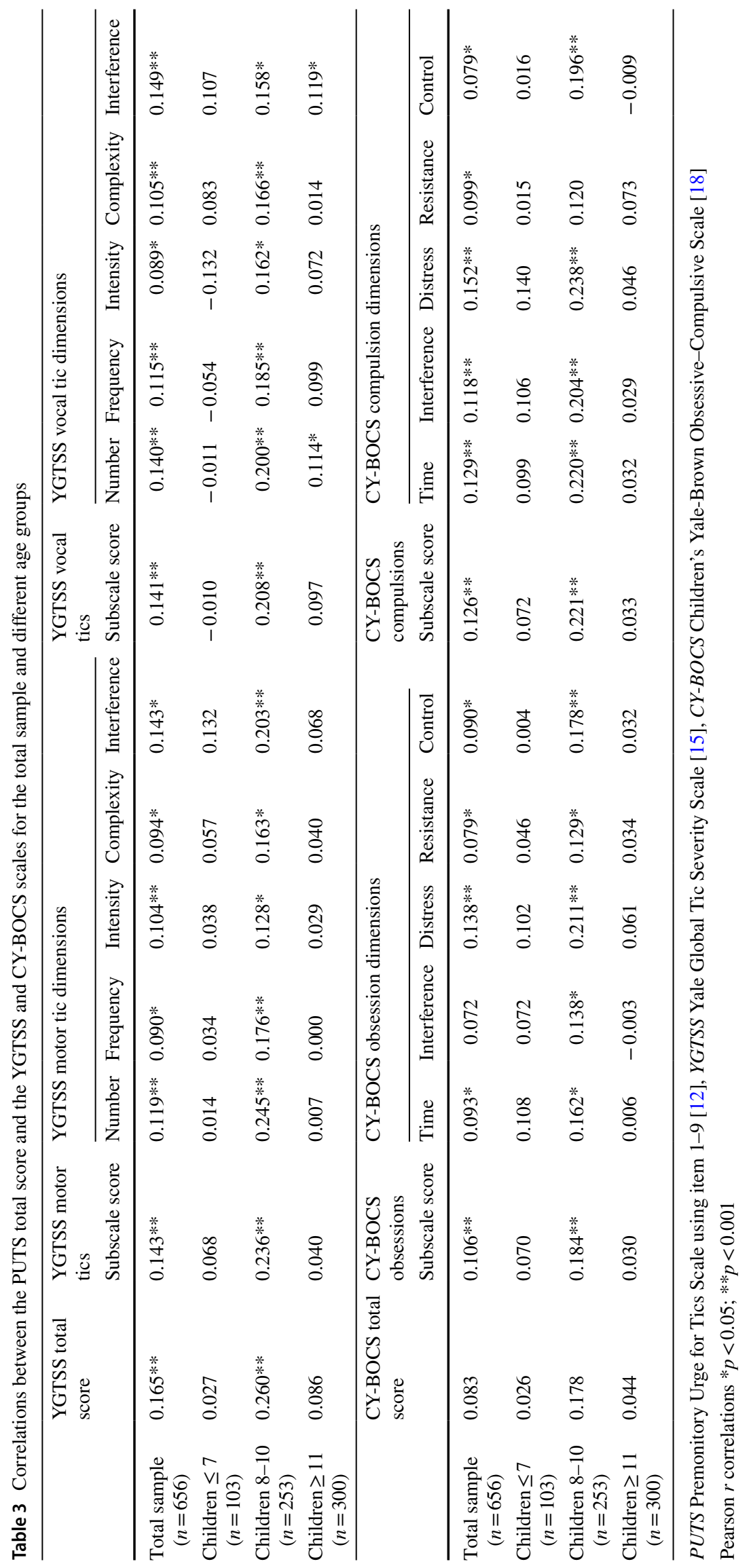


in children aged 8-10, but not in younger or older children. Other correlations did not yield significant results.

\section{Exploratory factor analysis}

See Table 4 for the factor loadings of the PUTS for the total sample, and divided by the three age groups (and Supplementary Table S2f for the factor loadings of the PUTS for children $\leq 10$ years). The inter-item correlation matrix (Supplementary Table S2e) showed good factorability of the PUTS in all groups, except for item 1, which was removed from the factor analysis in all groups due to multiple low inter-itemcorrelations $(r<0.20)$. Similarly, for children $\geq 11$ years, items 2 and 9 were removed (Supplementary Tables S2e, S2f). There was no multicollinearity between PUTS items.

After removing item 1 from the respective groups, the $\mathrm{KMO}$ for the total sample and all age groups was above the recommended value of 0.6 indicating sufficient sampling adequacy (Table 4 [34]). Furthermore, Bartlett's test of sphericity was significant for all groups [i.e., the total sample: $\chi^{2}(28)=1306.6, p<0.001$; children $\leq 7$ years $\chi^{2}(28)=207.7$ $p<0.001$; children between 8 and 10 years: $\chi^{2}(28)=535.8$, $p<0.001$; children $\leq 10$ years: $\chi^{2}(28)=751.5, p<0.001$; and children $\geq 11$ years: $\chi^{2}(15)=345.6, p<0.001$, respectively], indicating that correlations between items were sufficiently large to conduct an EFA.

An EFA with oblimin rotation across PUTS items 2-9 for the total sample indicated one factor (see Table 4). Initial eigenvalues demonstrated that this factor explained $42.2 \%$ of the variance. In the three-group analysis, an EFA across items $2-9$ for children $\leq 7$ years also revealed that all items loaded on one factor, explaining $47.4 \%$ of the variance (see Table 4), while it explained $43.7 \%$ for children between 8 and 10 years. In addition, in the two-group analysis, all items loaded on one factor for children $\leq 10$ years, explaining $44 \%$ of the variance (Supplementary Table S2f). However, an exploratory factor analysis for children $\geq 11$ years in both analyses revealed two factors, with a total explained variance of $67.9 \%$. Notably, the first factor that explained the most variance in the two-factorsolution included two OCD-related items (items 4 and 5). In children $\geq 11$ years, item 6 ('the feeling of an energy that needs to get out') had a communality score of .18, while in children $\leq 10$ years item 3 ('Right before I do a tic, I feel "wound up" or tense inside') had a communality score of .17, thus these items were subsequently removed from the respective factor analyses. Finally, after removing the two OCD-related items from all analyses, only one-factor solutions emerged for all groups. As a final remark, when repeating all analyses with the outliers included, all results remained similar.

\section{Discussion}

The present study investigated the psychometric properties of the PUTS in 656 children and adolescents aged $3-16$ years. Contrary to previous smaller sized studies [12, $16,17]$ that reported insufficient psychometric properties of the PUTS in children younger than 11 years, our results showed satisfactory reliability also in younger children. This suggests that the PUTS is suitable for children and adolescents across a broad age range. We found that the PUTS correlated significantly, yet weakly, with tic and OCD symptom severity, and with measures for ADHD and internalizing and externalizing behaviors, specifically in children between 8 and 10 years. These overall weak correlations point to different constructs as assessed by the PUTS and other scales measuring symptoms of different clinical diagnoses. While the PUTS was originally designed as a one-dimensional measure, we observed an underlying two-factor structure of the PUTS in children and adolescents above 10 years. This pointed to two distinct dimensions that are measured by the PUTS, of which one factor contained the two items that previously has been associated with OCD (i.e. 'the feeling that something is not "just right" or not complete'). Consistent with Woods et al. [12], PUTS item number 10 (measuring the ability to stop tics even if only for a short period of time) correlated less with the rest of the scale compared to the other items and, therefore, should not be used as part of the questionnaire for all age groups.

Internal reliability for all investigated age groups was in the good to excellent range. Previous authors explained their findings of low internal reliability of the PUTS in children of 11 and younger by difficulties in recognizing or articulating awareness of the urge $[12,16]$. It has also been suggested that perhaps the urges are not present during the initial stages of TS, but develop on average a few years after the first onset of tics, which usually is around 6 years of age [3, 10,38]. While our study confirms tic onset around 6 years of age, we also observed that 80 to $95 \%$ of the children of 10 years and younger experienced urges to some extent, which is more than previously reported in a large pediatric sample (47\% in children under the age of 10 [14]). Yet, our findings are similar to Woods et al. [12], who originally reported that all children of 10 years and younger experienced premonitory urges. Our study suggests that the presence of premonitory urges may already exist about the time tics develop and that urges can be reliably identified early in development. Additional support for the early presence of premonitory urges stems from the demonstrated efficacy of behavioral treatment focusing on premonitory urges in children under the age of 10 [39]. However, we did observe an age-dependent increased awareness of the premonitory urge across the age groups, with the youngest children reporting the least 


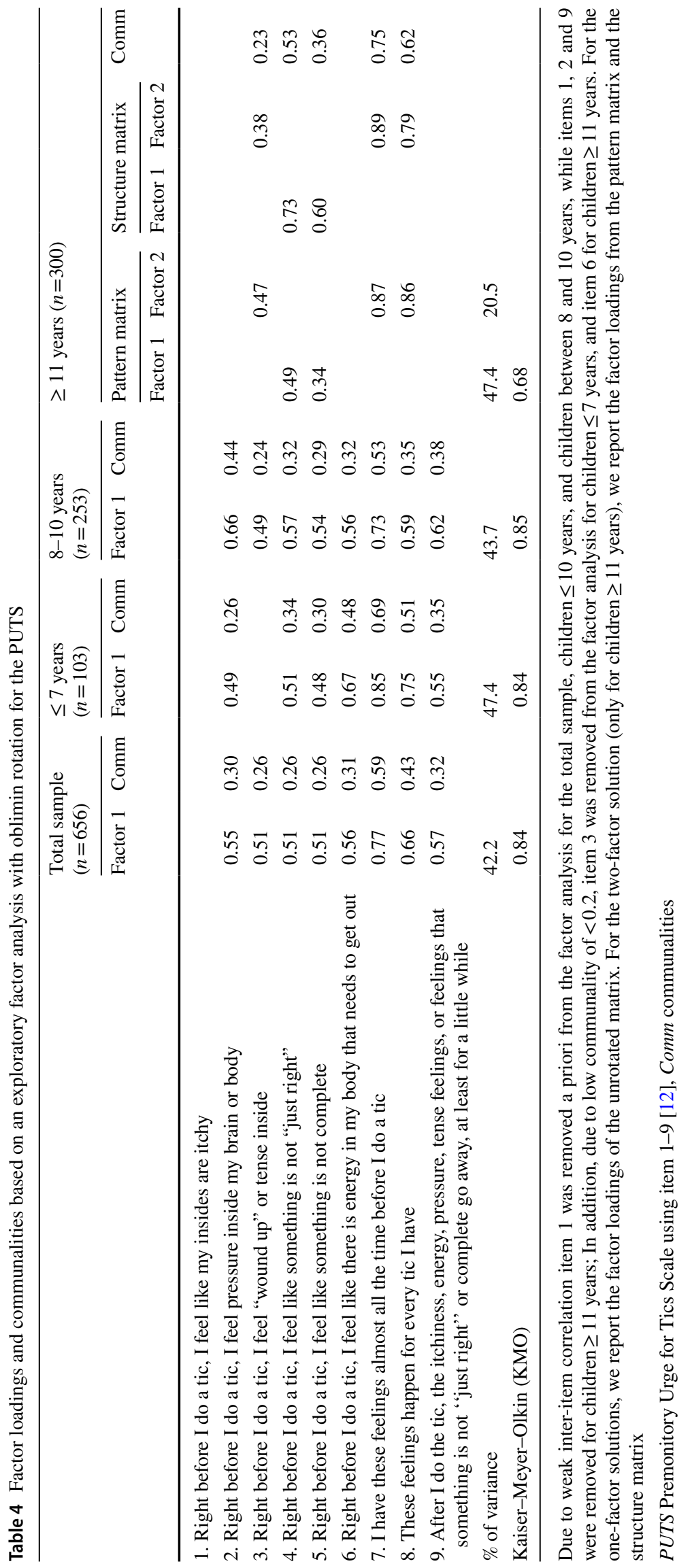


amount of urges (81\%) and the oldest participants the most $(97.5 \%)$. It remains questionable to what extent very young children are able to reliably fill in a self-report questionnaire. We cannot exclude that the parents have assisted in answering the PUTS items, even though there are reports of 5 -year-olds reliably filling in age-appropriate health-related questionnaires [40]. In sum, although our results point to a reliable use of the PUTS from young childhood well into adolescence, more research is warranted to further explore the possible existence and reporting of premonitory urges in very young children.

The weak, and largely non-existent correlations between the PUTS and tic severity as assessed by (subscales of) the YGTSS were unexpected. If tics are indeed semi-voluntary responses to premonitory urges [23], which is also presumed by one of the most endorsed items of the PUTS in our study (i.e., item 9, 'the feelings go away after I do the tic'), then more severe urges would be expected to be related to more severe tics. Our results are in contrast to a recent meta-analysis observing a moderate correlation $(r=0.296)$ between the severity of premonitory urges and tic symptoms [44], although this was based on a small number of studies using relatively small samples $(n=40-122)$ across children and adults, which may have biased findings [41]. One explanation for the weak association between premonitory urges and tic severity in our study may be that the PUTS and YGTSS questionnaires are actually measuring different constructs relating to distinct phenomena. This is in line with Ganos et al. [42] who suggested distinct neurological pathways for premonitory urges, tic generation, and tic suppression; and that premonitory urges may not be the driving force behind tics [43]. A similar distinction has previously been mentioned by Brandt et al. [35], showing only a weak relationship between premonitory urges measured by a realtime urge monitor and tic frequency; a relation that even weakened during tic suppression, suggesting a decoupling of urges and tics. On another note, limitations of the PUTS have been recognized before (e.g. being designed as a unitary construct, and not allowing the respondent to distinguish between specific urges for different tics [45]), leading to the recent development of a new measure to assess premonitory urges (I-PUTS, [45]). However, more research is warranted to investigate the validity of this new measure in comparison with the PUTS. Regarding the age effects, perhaps younger children are less able to distinguish between urges and tics [10], whereas the ability to differentiate between these phenomena may become more pronounced with increasing age. In children and adolescents above 10 years on the other hand, more severe urges may not necessarily be accompanied by more severe tics, as indicated in our study by the disappearing relation between the severity of urges and tics, perhaps due to a better awareness of the urges.
Two items of the PUTS representing mental phenomena that may be considered part of the OCD spectrum (i.e., items 4 and 5 referring to feelings of not "just right" and not complete) largely drove the association for children between 8 and 10 years in our study; this may suggest that a relation between the PUTS and OCD symptoms is spurious. Mixed findings regarding associations between PUTS severity and OCD severity have been documented before [12, 17, 23], although these results were only found in children of 11 years and older and in adults, while the recent meta-analysis that included these studies indicated a moderate association between premonitory urges and obsessive-compulsive symptoms [44]. Why this association exclusively existed in children between 8 and 10 years in our study cannot be readily explained, as no differences in OCD symptom severity between the investigated age groups were observed. Perhaps children between 8 and 10 years, at an age when symptoms of OCD are typically developing [37], have difficulty differentiating between premonitory urges that are associated with tics and those associated with OCD symptoms, which may become easier with increasing age [36]. Alternatively, as the frequency of these two mental urges appeared to slightly increase with age, so did other items captured by the PUTS, possibly outweighing the influence of these OCD-like urges, explaining the lack of association between the PUTS and OCD symptoms in children of 11 years and older. Of note, even though the correlations between premonitory urges and OCD symptoms in children between 8 and 10 years were significant, they were small, similar to the other age groups, indicating a weak relationship. Further research is needed to elucidate the complex relationship between tic and OCDrelated urges across development.

Consistent with the original PUTS [12], we found a onefactor solution in children of 10 years and younger. Confirming recent studies in children and adults [16, 19], and in line with the above discussed results, we found support for a twofactor solution in children of 11 years and older. The first and most important factor, explaining the most variance, pointed to items that are typically associated with obsessive-compulsive symptoms [5], which suggest a distinction between sensory phenomena related to OCD and those related to tics. The second factor, which explained less variance, included items that addressed the 'frequency of urges' before a tic in children as of 11 years (i.e., 'if the feelings are present almost all the time before a tic' and if 'these feelings happen for every tic'). This is in line with Raines [16] and has similarities to Brandt et al.'s [19] second factor described as the 'overall intensity of urges'. In sum, the age-related differences we observed so far regarding the underlying structure (one versus two-factor solution) of the PUTS, and the various items that had to be removed from the analyses in the older age group may indicate that the questions of the PUTS may be differently perceived at various ages. 
A major strength of this study was the large sample size and wide age range, allowing us to explore age-dependency across a broad age range. Potential limitations were, first, the use of multiple clinical sites across Europe, reflecting possible site differences in scoring and clinical populations. By regressing out the effect of site per variable, we tried to account for this bias. In addition, clinical interviewers were regularly trained and standardization of the procedures was discussed bi-annually. Second, our sample showed a relatively low number of comorbid ADHD and OCD diagnoses compared to previous studies investigating the psychometric properties of the PUTS [12, 16, 17], perhaps indicating a less severely affected sample.

In conclusion, the PUTS questionnaire exhibits good internal reliability in children and adolescents, also in children under the age of 10, which is younger than previously thought. Our study indicates that premonitory urges appear to be present at an early age, possibly starting at the onset of tics in some children. The overall weak correlations between the PUTS and, respectively, YGTSS and CY-BOCS scores suggest that different constructs are measured by the respective scales, distinguishing between premonitory urges, tics, and obsessive-compulsive symptoms. The observed twofactor structure of the PUTS in children of 11 years and older indicates that two separate dimensions of premonitory urges are measured in this age group, distinguishing between sensory phenomena related to tics and mental phenomena as often found in OCD. The age-related differences observed in this study may indicate the need for the development of an age-specific questionnaire to asses urges. Future research should focus on a closer examination of the use of the PUTS across development and how well it captures possible agedependent differences in premonitory urges and the relation with tics and comorbid symptoms.

Acknowledgements The authors are deeply grateful to all children and their parents who willingly participated to make this research possible. This project has received funding from the European Union's Seventh Framework Programme for research, technological development and demonstration under Grant agreement no. 278367. This research was supported by Stiftung Immunität und Seele (Burger, Müller, Schnell); and the National Institute for Health Research Biomedical Research Centre at Great Ormond Street Hospital for Children NHS Foundation Trust and University College London (Heyman); and Deutsche Forschungsgemeinschaft (DFG): projects 1692/3-1, 4-1 and FOR 2698 (Münchau); We thank all colleagues at the various study centers who contributed to data collection: Julie E. Bruun, Judy Grejsen, Christine L. Ommundsen, Mette Rubæk (Capital Region Psychiatry, Copenhagen, Denmark); Stephanie Enghardt (TUD Dresden, Germany); Stefanie Bokemeyer, Christiane Driedger-Garbe, Cornelia Reichert (MHH Hannover, Germany); Jenny Schmalfeld (Lübeck University, Germany); Elif Weidinger (LMU Munich, Germany); Martin L. Woods (Evelina London Children's Hospital, United Kingdom); Susanne Walitza (University of Zurich, Switzerland); Franciska Gergye, Margit Kovacs, Reka Vidomusz (Vadaskert Budapest, Hungary); Silvana Fennig, Ella Gev, Matan Nahon, Danny Horesh, Chen Regev, Tomer Simcha, (Tel Aviv, Petah-Tikva, Israel); Mascha van den Akker, Els van den Ban, Sebastian F.T.M. de Bruijn, Nicole Driessen, Andreas Lamerz, Marieke Messchendorp, Judith J.G. Rath, Anne Marie Stolte, Nadine Schalk, Deborah Sival, Noor Tromp and the Stichting Gilles de la Tourette (UMCG Groningen, Netherlands); Maria Teresa Cáceres, Fátima Carrillo, Laura Vargas, Ángela Periañez Vasco (Seville, Spain); and all who may not have been mentioned.

EMTICS group authorship/appendix: EMTICS group members are Alan Apter ${ }^{1}$, Valentina Baglioni ${ }^{2}$, Juliane Ball ${ }^{3}$, Noa BenaroyaMilshtein $^{1}$, Benjamin Bodmer ${ }^{4}$, Emese Bognar ${ }^{5}$, Bianka Burger ${ }^{6,7}$, Judith Buse ${ }^{4}$, Francesco Cardona ${ }^{2}$, Marta Correa Vela ${ }^{8}$, Nanette M. Debes $^{10}$, Andrea Dietrich ${ }^{9}$, Maria Cristina Ferro ${ }^{11}$, Carolin Fremer ${ }^{12}$, Blanca Garcia-Delgar ${ }^{13}$, Mariangela Gulisano ${ }^{11}$, Annelieke Hagen ${ }^{14,15}$, Julie Hagstrøm ${ }^{16}$, Tammy J. Hedderly ${ }^{17}$, Isobel Heyman ${ }^{18}$, Pieter J. Hoekstra $^{9}$, Chaim Huyser ${ }^{14,15}$, Marcos Madruga-Garrido ${ }^{19}$, Anna Marotta $^{20}$, Pablo Mir ${ }^{8}$, Astrid Morer ${ }^{13,21,22}$, Norbert Müller ${ }^{6,7}$, Kirsten Müller-Vahl ${ }^{12}$, Alexander Münchau ${ }^{23}$, Peter Nagy ${ }^{5}$, Valeria Neri ${ }^{2}$, Thaïra J.C. Openneer ${ }^{9}$, Alessandra Pellico ${ }^{11}$, Kerstin J. Plessen ${ }^{16,24}$ Cesare Porcelli ${ }^{20}$, Marina Redondo ${ }^{13}$, Renata Rizzo ${ }^{11}$, Veit Roessner ${ }^{4}$, Daphna Ruhrman ${ }^{1}$, Jaana M.L. Schnell ${ }^{6}$, Paola Rosaria Silvestri ${ }^{2}$, Liselotte Skov ${ }^{10}$, Tamar Steinberg ${ }^{1}$, Friederike Tagwerker Gloor ${ }^{3}$, Zsanett Tárnok ${ }^{5}$, Jennifer Tübing ${ }^{24}$, Victoria L. Turner ${ }^{17}$, Frank Visscher ${ }^{25}$

${ }^{1}$ Child and Adolescent Psychiatry Department, Schneider Children's Medical Center of Israel, affiliated to Sackler Faculty of Medicine, Tel Aviv University, Petah-Tikva, Israel

${ }^{2}$ University La Sapienza of Rome, Department of Human Neurosciences, Rome, Italy

${ }^{3}$ Clinic of Child and Adolescent Psychiatry and Psychotherapy, University of Zurich, Zurich, Switzerland

${ }^{4}$ Department of Child and Adolescent Psychiatry, Faculty of Medicine of the TU Dresden, Dresden, Germany

${ }^{5}$ Vadaskert Child and Adolescent Psychiatric Hospital, Budapest, Hungary

${ }^{6}$ Department of Psychiatry and Psychotherapy, University Hospital, LMU Munich, Munich, Germany

${ }^{7}$ Marion von Tessin Memory-Zentrum gGmbH, Munich, Germany

${ }^{8}$ Unidad de Trastornos del Movimiento, Servicio de Neurología y Neurofisiología Clinica. Instituto de Biomedicina de Sevilla (IBiS), Hospital Universitario Virgen del Rocio/CSIC/Universidad de Sevilla, Seville, Spain

${ }^{9}$ University of Groningen, University Medical Center Groningen, Department of Child and Adolescent Psychiatry, Groningen, The Netherlands

${ }^{10}$ Paediatric Department, Herlev University Hospital, Herlev, Denmark

${ }^{11}$ Child Neuropsychiatry Section, Department of Clinical and Experimental Medicine, School of Medicine, Catania University, Catania, Italy

${ }^{12}$ Clinic of Psychiatry, Socialpsychiatry and Psychotherapy, Hannover Medical School, Hannover, Germany

${ }^{13}$ Department of Child and Adolescent Psychiatry and Psychology, Institute of Neurosciences, Hospital Clinic Universitari, Barcelona, Spain

${ }^{14}$ De Bascule, Academic Center for Child and Adolescent Psychiatry, Amsterdam, The Netherlands

${ }^{15}$ Academic Medical Center, Department of Child and Adolescent Psychiatry, Amsterdam, The Netherlands

${ }^{16}$ Child and Adolescent Mental Health Center, Mental Health Services, Capital Region of Denmark and University of Copenhagen, Copenhagen, Denmark

${ }^{17}$ Evelina London Children's Hospital GSTT, Kings Health Partners AHSC, London, UK

${ }^{18}$ Great Ormond Street Hospital for Children, and UCL Institute of Child Health, London, UK 
${ }^{19}$ Sección de Neuropediatría, Instituto de Biomedicina de Sevilla (IBiS), Hospital Universitario Virgen del Rocío/CSIC/Universidad de Sevilla, Seville, Spain

${ }^{20}$ Azienda Sanitaria Locale di Bari, Mental Health Department, Child and Adolescent Service of Bari Metropolitan Area, Bari, Italy

${ }^{21}$ Institut d'Investigacions Biomediques August Pi i Sunyer (IDIBAPS), Barcelona, Spain

${ }^{22}$ Centro de Investigacion en Red de Salud Mental (CIBERSAM), Instituto Carlos III, Madrid, Spain

${ }^{23}$ Institute of Neurogenetics, University of Lübeck, Lübeck, Germany

${ }^{24}$ Service of Child and Adolescent Psychiatry, Department of Psychiatry, University Medical Center, University of Lausanne, Lausanne, Switzerland

${ }^{25}$ Admiraal De Ruyter Ziekenhuis, Department of Neurology, Goes, The Netherlands

Funding Müller-Vahl received funding for research from the EU (FP7PEOPLE-2012-ITN No. 316978), the German Research Society (DFG: GZ MU 1527/3-1), the German Ministry of Education and Research (BMBF: 01KG1421), the National Institute of Mental Health (NIMH), GW, Almirall, Abide Therapeutics, and Therapix Biosciences, and consultant's honoraria from Abide Therapeutics, Fundacion Canna, and Therapix Biosiences.

\section{Compliance with ethical standards}

Conflict of interest On behalf of all other authors, the corresponding author declares that the other authors have no conflict of interest.

Open Access This article is distributed under the terms of the Creative Commons Attribution 4.0 International License (http://creativeco mmons.org/licenses/by/4.0/), which permits unrestricted use, distribution, and reproduction in any medium, provided you give appropriate credit to the original author(s) and the source, provide a link to the Creative Commons license, and indicate if changes were made.

\section{References}

1. American Psychiatric Association (2013) Diagnostic and statistical manual of mental disorders, 5th edn. American Psychiatric Press, Washington, DC

2. Hirschtritt ME, Lee PC, Pauls DL, Dion Y, Grados MA, Illmann C et al (2015) Lifetime prevalence, age of risk, and etiology of comorbid psychiatric disorders in Tourette syndrome. JAMA Psychiatry 72(4):325-333. https://doi.org/10.1001/jamapsychi atry.2014.2650.Lifetime

3. Leckman F, Walker E, Levi-Pearl S (1993) Premonitory urges in Tourette's syndrome. Am J Psychiatry 150(1):98-102

4. Cox JH, Seri S, Cavanna AE (2018) Sensory aspects of Tourette syndrome. Neurosci Biobehav Rev 88:170-176

5. Rajagopal S, Cavanna AE (2014) Premonitory urges and repetitive behaviours in adult patients with Tourette syndrome. J Neurol Sci 35:969-971. https://doi.org/10.1007/s10072-014-1706-8

6. Crossley E, Cavanna AE (2013) Sensory phenomena: clinical correlates and impact on quality of life in adult patients with Tourette syndrome. Psychiat Res 209(3):705-710. https://doi. org/10.1016/j.psychres.2013.04.019

7. Eddy CM, Rizzo R, Gulisano M, Agodi $\mathrm{A}$, Barchitta $\mathrm{M}$, Calì $\mathrm{P}$ et al (2011) Quality of life in young people with Tourette syndrome: a controlled study. J Neurol 258(2):291-301. https://doi. org/10.1007/s00415-010-5754-6
8. Dutta N, Cavanna AE (2013) The effectiveness of habit reversal therapy in the treatment of Tourette syndrome and other chronic tic disorders: a systematic review. Funct Neurol 28(1):7-12

9. Van de Griendt JMTM, Verdellen CWJ, van Dijk MK, Verbraak MJPM (2013) Behavioural treatment of tics: habit reversal and exposure with response prevention. Neurosci Biobehav Rev 37(6):1172-1177. https://doi.org/10.1016/j.neubi orev.2012.10.007

10. Banaschewski T, Rothenberger A (2003) Premonitory sensory phenomena and suppressibility of tics in Tourette syndrome: developmental aspects in children and adolescents. Dev Psychopathol 45:700-703. https://doi.org/10.1017/S0012162203001294

11. Cavanna AE, Black KJ, Hallet M, Voon V (2017) Neurobiology of the premonitory urge in Tourette syndrome: Pathophysiology and treatment implication. J Neuropsychiatry Clin Neurosci 29(2):95-104. https://doi.org/10.1176/appi.neuropsych.16070141

12. Woods DW, Piacentini J, Himle MB, Chang S (2005) Premonitory Urge for Tics Scale (PUTS): initial psychometric results and examination of the premonitory urge phenomenon in youths with tic disorders. J Dev Behav Pediatr 26(6):397-403. https://doi. org/10.1097/00004703-200512000-00001

13. American Psychiatric Association (2000) Diagnostic and statistical manual of mental disorders, 4th edn. American Psychiatric Press, Washington, DC

14. Sambrani T, Jakubovski E, Muller-Vahl K (2016) New insights into clinical characteristics of Gilles de la Tourette syndrome: findings in 1032 patients from a single German Center. Front Neurosci 10(9):415. https://doi.org/10.3389/fnins.2016.00415

15. Leckman JF, Riddle MA, Hardin MT, Ort SL, Swartz KL, Stevenson J, Cohen DJ (1989) The Yale global tic severity scale: initial testing of a clinician-rated scale of tic severity. J Am Acad Child Adolesc Psychiatry 28:566-573

16. Raines JM, Edwards KR, Sherman MF, Higginson CI, Winnick JB, Navin K et al (2017) Premonitory Urge for tics scale (PUTS): replication and extension of psychometric properties in youth with chronic tic disorders (CTDs). J Neural Transm 125(4):727-734. https://doi.org/10.1007/s00702-017-1818-4

17. Steinberg T, Shmuel Baruch S, Harush A, Dar R, Woods D, Piacentini J, Apter A (2010) Tic disorders and the premonitory urge. J Neural Transm 117(2):277-284. https://doi.org/10.1007/s0070 2-009-0353-3

18. Scahill L, Riddle MA, McSwiggin-Hardin M, Ort SI, King RA, Goodman WK et al (1997) Children's Yale-Brown obsessive compulsive scale: reliability and validity. J Am Acad Child Adolesc Psychiatry 36(6):844-852

19. Brandt VC, Beck C, Sajin V, Anders S, Münchau A (2016) Convergent validity of the PUTS. Front Psychiatry 7(4):1-7. https:// doi.org/10.3389/fpsyt.2016.00051

20. Leckman JF, Zhang H, Vitale A et al (1998) Course of tic severity in Tourette syndrome: the first two decades. Pediatrics 102:14-19

21. Eddy CM, Cavanna AE (2014) Premonitory urges in adults with complicated and uncomplicated Tourette syndrome. Behav Modif 38(2):264-275. https://doi.org/10.1177/0145445513504432

22. Schrag A, Martino D, Apter A, Ball J, Bartolini E, BenaroyaMilshtein N et al (2019) European Multicentre tics in children studies (EMTICS): protocol for two cohort studies to assess risk factors for tic onset and exacerbation in children and adolescents. Eur Child Adolesc Psychiatry 28(1):91-109. https://doi. org/10.1007/s00787-018-1190-4

23. Reese HE, Scahill L, Peterson AL, Crowe K, Woods DW, Piacentini J et al (2014) The premonitory urge to tic: measurement, characteristics, and correlates in older adolescents and adults. Behav Ther 45(2):177-186. https://doi.org/10.1016/j.beth.2013.09.002

24. Storch EA, Murphy TK, Adkins JW, Lewin AB, Geffken GR, Johns NB et al (2006) The children's Yale-Brown obsessivecompulsive scale: psychometric properties of child- and 
parent-report formats. J Anxiety Disord 20(8):1055-1070. https ://doi.org/10.1016/j.janxdis.2006.01.006

25. Swanson JM (1992) School-based assessments and interventions for ADD students. K. C. Publishing, Irvine

26. Bussing R, Fernandez M, Harwood M, Wei Hou W, Garvan CW, Eyberg SM et al (2008) Parent and teacher SNAP-IV ratings of attention deficit/hyperactivity disorder symptoms: psychometric properties and normative rating from a school district sample. Assessment 15(3):317-328. https://doi.org/10.1177/1073191107 313888

27. Ehlers S, Gillberg C, Wing L (1999) A screening questionnaire for Asperger syndrome and other high-functioning autism spectrum disorders in school age children. J Autism Dev Disord 29(2):129-141

28. Goodman R (1997) The Strengths and difficulties questionnaire: a research note. J Child Psychol Psychiatry 38(5):581-586. https ://doi.org/10.1111/j.1469-7610.1997.tb01545.x

29. Kline P (1986) A handbook of test construction: introduction to psychometric design. Methuen \& Co, New York

30. Streiner DL (2003) Being inconsistent about consistency: when coefficient alpha does and does not matter. Assessment $80: 217-222$

31. Cohen J (1992) A power primer. Psychol Bull 112(1):155-159

32. Watson JC (2017) Establishing evidence for internal structure using exploratory factor analysis. Meas Eval Couns Dev 50(4):232-238. https://doi.org/10.1080/07481756.2017.1336931

33. Field A (2013) Discovering statistics using IBM SPSS. Sage Publication Ltd, Thousand Oaks

34. Kaiser HF, Rice J (1974) Little Jiffy, Mark IV. Educ Psychol Meas 34:111-117

35. Brandt VC, Beck C, Sajin V, Baaske MK, Bäumer T, Beste C, Anders S, Münchau A (2016) Temporal relationship between premonitory urges and tics in Gilles de la Tourette syndrome. Cortex 77:24-37

36. Rozenman M, Johnson OE, Chang SW, Woods DW, Walkup JT, Wilhelm S, Peterson A, Scahill L, Piacentini J (2015) Relationships between premonitory urge and anxiety in youth with chronic tic disorders. J Child Health Car 44:235-248
37. Masi G, Millepiedi S, Mucci M, Milantoni L, Arcangeli F (2005) A naturalistic study of referred children and adolescents with obsessive-compulsive disorder. J Am Acad Child Adolesc Psychiatry 44(7):673-681

38. Bloch MH, Leckman JF (2009) Clinical course of Tourette syndrome. J Psychosom Res 67(6):497-501

39. Piacentini JC, Woods DW, Scahill LN, Wilhelm S, Peterson AL, Chang S et al (2010) Behavior therapy for children with Tourette disorder: a randomized controlled trial. JAMA 303(19):19291937. https://doi.org/10.1001/jama.2010.607

40. Varni JW, Limbers CA, Burwinkle TM (2007) How young can children reliably and validly self-report their health-related quality of life? An analysis of 8591 children across age subgroups with the PedsQL ${ }^{\mathrm{TM}} 4.0$ Generic core scales. Health Qual Life Outcomes 5:1-13. https://doi.org/10.1186/1477-7525-5-1

41. Anthoine E, Moret L, Regnault A, Sbille V, Hardouin J-B (2014) Sample size used to validate a scale: a review of publications on newly-developed patient reported outcomes measures. Health Qual Life Outcomes 12:176. https://doi.org/10.1186/s1295 5-014-0176-2

42. Ganos C, Kahl U, Schunke O, Kühn S, Haggard P, Gerloff C et al (2012) Are premonitory urges a prerequisite of tic inhibition in Gilles de la Tourette syndrome? J Neurol Neurosurg Psychiatry 83:975-978. https://doi.org/10.1136/jnnp-2012-30303343

43. Ganos C, Garrido A, Navalpotro-Gomez I, Ricciardi L, Martino $\mathrm{D}$, Edwards MJ et al (2015) Premonitory urge to tic in tourette's is associated with interoceptive awareness. Mov Disord 30(9):1198 1202. https://doi.org/10.1002/mds.26228

44. Li Y, Wang F, Liu J, Wen F, Yan C, Zhang J, Lu X, Cui Y (2019) The correlation between the severity of premonitory urges and tic symptoms: a meta-analysis. J Child Adolesc Psychopharmacol. https://doi.org/10.1089/cap.2019.0048 (Epub ahead of print)

45. McGuire JF, McBride N, Piacentini J, Johnco C, Lewin AB, Murphy TK, Storch EA (2016) The premonitory urge revisited: an individualized premonitory urge for tics scale. J Psychiatr Res 83:176-183 